\title{
Shakespeare im deutschsprachigen Musiktheater des 18. und 19. Jahrhunderts
}

Andreas Waczkat (Universität Göttingen)

Der Versuch eines Überblicks über die Adaptionen von Shakespeares Dramen im deutschsprachigen Musiktheater, wie er hier unternommen werden soll, birgt zahlreiche Herausforderungen in sich: herausfordernd ist die teilweise sehr schwierige Quellenlage, herausfordernd ist die gattungstheoretische Bandbreite der Adaptionen, herausfordernd ist der nicht eben komfortable Forschungsstand, ${ }^{1}$ herausfordernd ist es auch, den historischen Querbalken in zwei Jahrhunderten aufzulegen, selbst wenn der im Folgenden betrachtete Zeitraum durch die faktischen Zeugnisse bestimmt genau genommen nur wenig mehr als ein Jahrhundert umfasst, nämlich die Zeit von den 1760er- bis zu den 1870er-Jahren. Diese historische Spanne reicht somit von den Opernreformen der zweiten Hälfte des 18. Jahrhunderts bis hin zu einer Zeit, da die Musikdramen Richard Wagners das Bild des deutschsprachigen Musiktheaters bestimmen.

Im Umfeld der Opernreformen der zweiten Hälfte des 18. Jahrhunderts werden zahllose Spielarten der Verbindung von Musik, dramatischen Texten und

\footnotetext{
${ }^{1}$ Einschlägig ist zunächst Dieter Martin, „Deutsche Shakespeare-Opern um 1800 (08.10.2005)“, in: Goethezeitportal, http://www.goethezeitportal.de/db/wiss/epoche/martin_shakespeare_opern.pdf (zuletzt aufgerufen am 30.05.2015). Eine Übersicht von Vertonungen in verschiedenen Gattungen gibt Joachim Draheim, „Shakespeare“, in: Ludwig Finscher (Hrsg.), Die Musik in Geschichte und Gegenwart. Zweite neubearbeitete Ausgabe, Personenteil 15, Kassel: Bärenreiter 2006, Sp. 660-671, hier Sp. 667-669.
} 
Bühnenaktion sowohl theoretisch diskutiert als auch praktisch ausprobiert. ${ }^{2}$ Die Bandbreite reicht dabei von den Opern Christoph Willibald Glucks, die nach einer Reform der opera seria innerhalb der Möglichkeiten und Grenzen der eigenen Gattung streben, über die musikalischen Dramen Johann Heinrich Rolles, die man sich am ehesten als Opern vorstellen kann, die gezielt für eine konzertante, also ohne Bühnenaktion gestaltete Aufführung konzipiert sind, bis hin zu neu entworfenen Gattungen wie dem Melodram, das im deutschsprachigen Raum zuerst bei Jiři Antonín Benda in den 1760er-Jahren eine Blütezeit erlebt und, im Gegensatz zu vielen anderen Versuchen dieser Zeit, auch in der Folgezeit nicht völlig aus dem Repertoire des musikdramatischen Komponierens verschwindet.

Das zeitliche Zusammentreffen dieser Opernreformen mit dem Erscheinen der Dramen Shakespeares in deutscher Übersetzung hat die Verwendung von Shakespeares Texten im deutschsprachigen Musiktheater zweifellos mehr als nur begünstigt. Vielmehr scheint es, als hätten Shakespeares Dramen per se etwas Neuartiges angeboten, nach dem die Opernreformer in der Abkehr vom metastasianischen Typus der opera seria mit seiner stereotypen Ordnung von zumeist drei Personenpaaren, der meist ebenso stereotypen Verflechtung politischer und erotischer Intrigen und der als künstlich empfundenen Trennung von Rezitativen und Arien gesucht, das deutschsprachige Dichter aber weniger erfolgreich angeboten haben. ${ }^{3}$ Und so dürfte es auch kein Zufall sein, dass im Netzwerk der Opernreformer - mit Ausnahme Glucks - und Operreformen auch die frühesten Versuche lokalisiert sind, Shakespeare für die Opernbühne zu entdecken. Dabei haben sich freilich zwei schwer wiegende Probleme gezeigt, für die es unterschiedliche Lösungsansätze gegeben hat: das eine Problem ist der Umfang der Shakespearschen Dramentexte, der einer vollständigen musikdramatischen Umsetzung entgegensteht, das andere ist der zumindest außerhalb der Lustspiele tragische Ausgang der Dramen, für den auch im Kontext der verschiedenen Opernreformen der Boden im Musiktheater noch nicht bereitet gewesen ist. ${ }^{4}$ Das lieto fine, das im

\footnotetext{
${ }^{2}$ Silke Leopold und Michael Zimmermann, „Opernreformen“, in: Carl Dahlhaus (Hrsg.), Die Musik des 18. Jahrhunderts (Neues Handbuch der Musikwissenschaft; 5), Laaber: Laaber-Verlag 1985, S. 239-253; Arnold Jacobshagen, „Opernkritik und Opern-,Reform", in: Herbert Schneider und Siegfried Wiesend (Hrsg.), Die Oper im 18. Jahrhundert (Handbuch der musikalischen Gattungen; 12), Laaber: Laaber-Verlag 2001, S. 74-84.

${ }^{3}$ Bemerkenswert ist in diesem Zusammenhang der namentlich nicht gekennzeichnete Eintrag „Oper“ in: Johann Georg Sulzer, Allgemeine Theorie der schönen Künste, zweite vermehrte Auflage Leipzig: Weidmann 1792-1794, Bd. III, S. 579, in dem die epischen Bardiete Friedrich Gottlieb Klopstocks als Textvorlagen „für eine völlig neue Art des Dramas“ favorisiert werden. Einen nicht erhalten gebliebenen Versuch hat dazu offenbar einzig Christoph Willibald Gluck mit einer Vertonung von Szenen aus Klopstocks Hermannschlacht unternommen. Eine Notiz dazu findet sich allein bei Klopstock selbst, und es ist unklar, ob Gluck vielleicht nur die Möglichkeit einer Vertonung erwogen hat.

${ }^{4}$ Martin, „Shakespeare-Opern“ (wie Anm. 1), S. 13.
} 
metastasianischen Operntypus noch normativ ist, wird zwar nicht mehr durchgehend als verbindlich angesehen, bleibt aber dennoch zumindest auf einer MetaEbene das Ziel. Wenn beispielshalber am Ende von Rolles 1779 uraufgeführtem musikalischen Drama Thirza und ihre Söhne nach einem Libretto August Hermann Niemeyers nicht nur alle sieben Söhne der Protagonistin den Tod finden, sondern diese auch selbst noch den Freitod wählt - alles übrigens getreu der von Niemeyer bearbeiteten biblischen Vorlage im zweiten Buch der Makkabäer -, dann scheint dies nur möglich, indem der Ausgang der Handlung einerseits christlichkathartisch umgedeutet und andererseits mit der - biblisch nicht mehr bezeugten Bekehrung des syrischen Peinigers Chryses als christliches Heilsgeschehen positiv ausgedeutet wird. ${ }^{5}$

Einer der Lösungsansätze für das Problem der Textmenge besteht darin, Shakespeares Dramentexte nur in Ausschnitten überhaupt zu vertonen. Ein bemerkenswertes Beispiel dazu und gleichzeitig die vermutlich früheste Shakespeare-Vertonung in deutscher Sprache überhaupt stammt allerdings nicht aus dem eigentlichen Bereich des deutschsprachigen Musiktheaters, sofern wir es mit der Vorstellung einer Bühnenhandlung verbinden. Es handelt sich vielmehr um eine Verbindung von Ausschnitten, oder besser: Versatzstücken aus dem Hamlet-Monolog mit einer der freien Clavierfantasien von Carl Philipp Emanuel Bach, und so ist die Bühne, auf der Hamlet auftritt, sehr klein: Es ist das Wohnzimmer von Heinrich Wilhelm von Gerstenberg in Lyngby bei Kopenhagen. ${ }^{6}$ Aber es ist gleichzeitig eine für die Anfangszeit der Rezeption der Shakespearschen Dramen in deutscher Sprache sehr typische Bühne. Sie führt in das Umfeld der anwachsenden und gleichzeitig nach der eigenen Identität noch suchenden deutschen Bürgerlichkeit, in das Umfeld des protestantischen Nordens und der Mitte des deutschsprachigen Raums, in dem man durch den Erwerb von humanistischer Bildung nach einer Art geistigen Adels strebte und auf diese Weise den Gedanken eines Bildungsbürgertums begründete, ohne dieses auch nur im Entferntesten so benennen zu wollen oder zu können. ${ }^{7}$

Die Dramen Shakespeares passen perfekt in dieses Ideal, und ihre Rezeption in deutscher Sprache zeigt das nahezu idealtypisch. Zu den ersten Übersetzern

\footnotetext{
${ }^{5}$ Andreas Waczkat, Johann Heinrich Rolles musikalische Dramen. Theorie, Werkbestand und Überlieferung einer Gattung im Kontext bürgerlicher Empfindsamkeit, Beeskow: ortus 2007, S. 235-238.

${ }^{6}$ Arnfried Edler, „Carl Philipp Emanuel Bachs Wirkung auf das Musikleben seiner Zeit“, in: Carl Philipp Emanuel Bach. Musik und Literatur in Norddeutschland (Schriften der SchleswigHolsteinischen Landesbibliothek; 4), Heide: Westholsteinische Verlagsanstalt 1988, S. 20-39, hier bes. S. 30-31 und 36.

7 Wolfgang Kaschuba, „Deutsche Bürgerlichkeit nach 1800. Kultur als symbolische Praxis“, in: Jürgen Kocka (Hrsg.), Bürgertum im 19. Jahrhundert. Deutschland im europäischen Vergleich, Bd. II: Wirtschaftsbürger und Bildungsbürger, Göttingen: Vandenhoeck und Ruprecht 1995, S. 92-127, hier S. $112 \mathrm{f}$.
} 
gehören der Pfarrerssohn Christoph Martin Wieland, der studierte Theologe und spätere Professor für englische Sprache am Braunschweiger Collegium Carolinum Johann Joachim Eschenburg, eine Generation später dann August Wilhelm Schlegel, wie Wieland ein Pfarrerssohn, und Ludwig Tieck, der literarisch ebenso ambitionierte wie begabte Sohn eines Berliner Seilermeisters. Auch Gerstenberg begeisterte sich in einem ähnlichen Kontext für die Dramen Shakespeares, die er in Wielands Übersetzung kennen gelernt und in Rezensionen hoch gelobt hatte. Geboren 1737 in Tondern als Sohn eines Rittmeisters in dänischen Diensten, besuchte er seit 1751 das Christianeum in Altona, studierte Fremdsprachen und deren Literatur. Ein Jura-Studium an der Universität Jena brach er nach zwei Jahren ab und trat 1760 in die dänische Armee ein. Seit 1765 lebte Gerstenberg mit seiner Familie in Lyngby bei Kopenhagen und widmete sich immer stärker der Literatur, die ihn auch zuvor schon ausführlich beschäftigt hatte: als Autor, aber auch als Übersetzer, Rezensent und Herausgeber. In seinem Haus fand sich rasch ein Kreis Interessierter zusammen, in dem neben der Literatur besonders auch die Musik wesentlich war; zusammen mit seiner Ehefrau Sophie Trochmann veranstaltete Gerstenberg regelmäßige Hauskonzerte ${ }^{8}$ und trat selber auch als Librettist in Erscheinung. 1765 erschien seine Kantate Ariadne auf Naxos im Druck $^{9}$, die immerhin vier Mal vertont worden ist: von Johann Adolph Scheibe ${ }^{10}$, Johann Christoph Friedrich Bach ${ }^{11}$, Jiři Antonín Benda ${ }^{12}$ und Johann Friedrich Reichardt ${ }^{13}$, wobei namentlich die Arbeiten von Scheibe und Benda sehr deutlich auf das Umfeld der Opernreformen verweisen. In die Zeit in Lyngby fällt auch Gerstenbergs intensive Rezeption der Dramen Shakespeares, die er in den Schleswiger Litteraturbriefen der Jahre 1766, 1767 und 1770 wie auch in seinen Briefen über Merkwürdigkeiten der Literatur behandelte und sich dabei sowohl mit Shakespeares Texten wie auch deren deutschen Übersetzungen beschäftigte. ${ }^{14} 1768$

\footnotetext{
8 Karl-Maria Guth, „Biographie“, in: Heinrich Wilhelm von Gerstenberg, Briefe über Merkwürdigkeiten der Literatur, hrsg. von Karl-Maria Guth, Berlin: Hofenberg 2013, S. 288.

${ }^{9}$ Heinrich Wilhelm von Gerstenberg, Ariadne auf Naxos. Eine Kantate, s.l.: s.n. 1765.

${ }^{10}$ Johann Adolph Scheibe, Tragische Kantaten für eine oder zwo Singestimmen und das Clavier: Nämlich: des Herrn von Gerstenbergs Ariadne auf Naxos, und Johann Elias Schlegels Prokris und Cephalus. In die Musick gesetzt, und nebst einem Sendschreiben, worinnen vom Recitativ überhaupt und von diesen Kantaten insonderheit geredet wird, Kopenhagen und Leipzig: Mumme 1765.

${ }^{11}$ Dies lässt sich, obwohl die Musik nicht erhalten ist, vermuten anhand eines Textbuchs: Ariadne auf Naxos. Eine Kantate vom Herrn von Gerstenberg mit Veränderungen aus einem Briefe des Verfassers, hrsg. von Johann Christoph Friedrich Bach, Lemgo: Meyer 1774.

12 Jiři Antonín Benda, Klavierauszug von Ariadne auf Naxos, einem Duodrama, Leipzig: Schwickert 1778. Der Text ist hier von Johann Christian Brandes etwas eingerichtet worden.

${ }^{13}$ Ariadne auf Naxos. Ein Singstück von Herrn von Gerstenberg, in Musik gesetzt von Herrn Kapellmeister Reichardt, Erfurt: Görling 1780.

14 Shakespeare-Rezeption: Die Diskussion um Shakespeare in Deutschland, hrsg. von Hansjürgen Blinn, Berlin: Erich Schmidt Verlag 1982, Bd. 1, S. 24-27.
} 
schließlich entstand der Hamlet-Monolog, der wohl in Gerstenbergs hausmusikalischem Kreis verortet ist - möglicherweise von Gerstenberg selbst gesungen, am Clavichord begleitet von seiner Frau.

Nun ist dieser Monolog freilich mehr als merkwürdig ausgefallen. Schon der nur im Vorwort abgedruckte - Titel in der von Carl Friedrich Cramer herausgegebenen Sammlung Flora ${ }^{15}$ weist auf die Konzeption hin: Phantasie von C. P. E. Bach, mit doppelt unterlegtem Text von Gerstenberg. ${ }^{16}$ Gerstenberg hat sich folglich einer Musik bedient, die aus einem gänzlich anderen Kontext stammt. Der Klaviersatz nämlich ist die 1753 gedruckte freie Fantasie in $c$-Moll von Carl Philipp Emanuel Bach ${ }^{17}$, der Gerstenberg den stark gekürzten Monolog unterlegt hat, mit einer Melodie, die überwiegend aus der Oberstimme des Klaviersatzes herausgelöst ist:

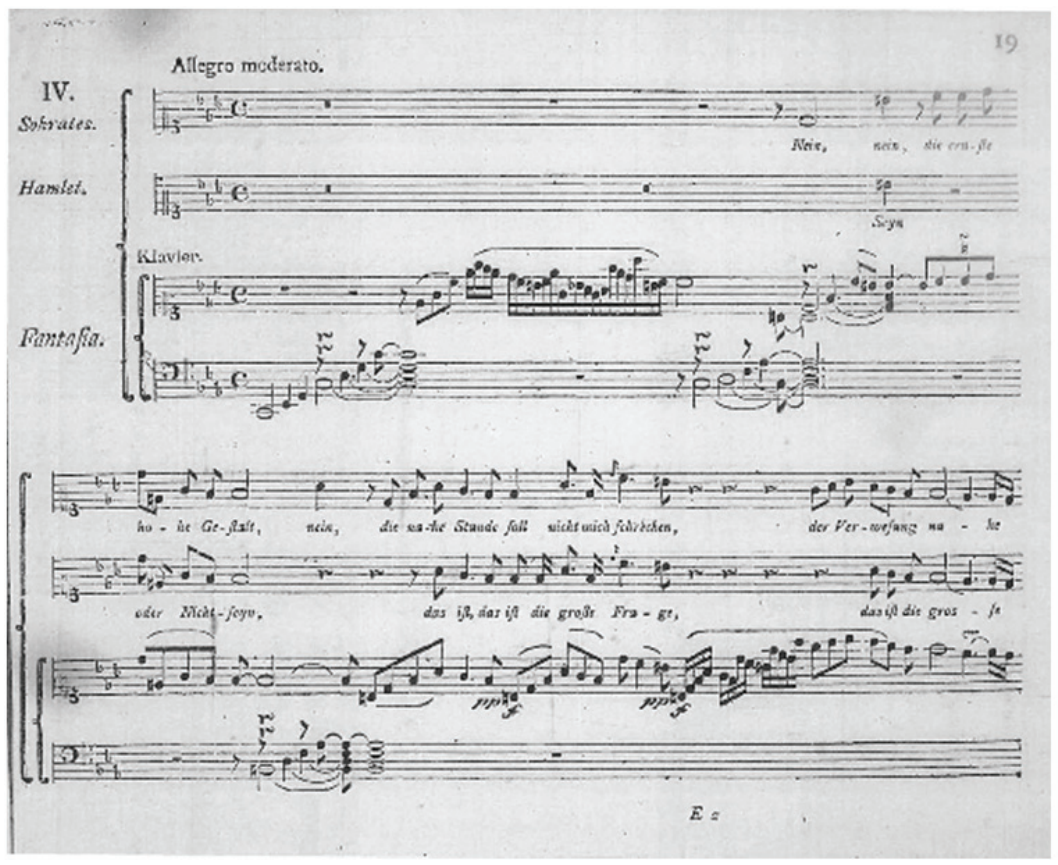

Flora. Erste Sammlung enthaltend: Compositionen für Gesang und Clavier, Kiel 1787, S. 19. Die Seite zeigt den Anfang von Carpl Philipp Emanuel Bachs Fantasie mit den beiden alternativen von Gerstenberg hinzugefügten Texten

15 Flora. Erste Sammlung [mehr nicht erschienen] enthaltend: Compositionen für Gesang und Klavier von Gräven, Gluck, Bach, Adolph Kunzen, F. L. Æ. Kunzen, Reichardt, Schwanenberger. Herausgegeben von C. F. Cramer, Kiel: [Selbstverlag] 1787.

${ }^{16}$ Enthalten in: Flora. Erste Sammlung enthaltend Compositionen für Gesang und Clavier, hrsg. von Carl Friedrich Cramer, Kiel: [Selbstverlag] 1787, S. 19-27.

${ }^{17}$ Enthalten in dessen Exempeln nebst achtzehn Probe-Stücken in sechs Sonaten zum [...] Versuch über die wahre Art das Clavier zu spielen, Berlin: [Selbstverlag] 1753, S. $19 \mathrm{f}$. 
Gerstenberg expliziert mit dieser Textunterlegung das, was er selbst als den musikalischen Gehalt dieser Instrumentalmusik empfunden hat. Seiner Ansicht nach ist das offenbar die Nähe zum edlen Selbstmord. Denn noch 20 Jahre später unterlegt er der Fantasie ein zweites Mal einen Text, dieses Mal einen eigenen: einen Monolog des Sokrates, der im Begriff steht, den Giftbecher zu trinken und wie Hamlet über das lichtvolle Weiterleben der Seele nach dem Tod im dunklen Grab sinniert. Ohne dass dies angesprochen würde, ist darin doch die christliche Heilserwartung ebenfalls unverkennbar.

Carl Philipp Emanuel Bach war eine solche konkrete Bedeutungszuschreibung an seine freie Fantasie suspekt. Er hielt von Gerstenbergs Einrichtung nichts und sperrte sich lange gegen eine Veröffentlichung. ${ }^{18}$ Doch das ist wohl nicht der einzige Grund, warum Gerstenbergs Einrichtung bis heute bestenfalls als Kuriosum einer musikhistorischen Übergangsepoche zur Kenntnis genommen wird. Hat Laurenz Lütteken auch sehr umfangreich herausgearbeitet, wie sehr das Monologische als Denkform die Musik dieser Zeit prägt, ${ }^{19}$ bleibt doch die tatsächliche monologische Rezitation der Gattung des Melodrams vorbehalten, in dem der Text in theatralischer Sprache vorgetragen, aber nicht metrisch gebunden gesungen wird.

Auch viele andere Bearbeitungen von Shakespeares Werken für die deutschsprachige Opernbühne haben bei aller Begeisterung für seine Dramen den Weg in das Repertoire nicht gefunden. Jiři Antonín Bendas Romeo und Julie, entstanden 1776 in Gotha ${ }^{20}$ nach dem Text von Friedrich Wilhelm Gotter ${ }^{21}$ ist zwar als Singspiel im 18. Jahrhundert recht populär gewesen, hat aber schon das 19. Jahrhundert nicht mehr erreicht. Lässt man Felix Mendelssohn Bartholdy Schauspielmusik zu Ein Sommernachtstraum einmal außer Acht, sind es danach lediglich Otto Nicolais Die lustigen Weiber von Windsor aus dem Jahr 1849 und Hermann Goetz' Der Widerspenstigen Zähmung aus dem Jahr 1874, die mehr als nur kurzfristig gespielt worden sind. Richard Wagners frühe Oper Das Liebesverbot oder Die Novize von Palermo, eher frei nach Shakespeares Maß für Maß, uraufge-

\footnotetext{
${ }^{18}$ Edler, „Bachs Wirkung“ (wie Anm. 6), S. 29.

19 Laurenz Lütteken, Das Monologische als Denkform in der Musik zwischen 1760 und 1785 (Wolfenbütteler Studien zur Aufklärung; 24), Tübingen: Niemeyer 1998.

${ }^{20}$ Jiři Antonín Benda, Klavierauszug von Romeo und Julie, einer Oper in drey Akten, in Musik gesetzt, Leipzig: Dyk 1778. Die Uraufführung fand am 25. September 1776 im Herzoglichen Hoftheater Gotha statt, ein Autograph der Partitur sowie ein Theaterzettel der Uraufführung befinden sich in der Staatsbibliothek Preußischer Kulturbesitz Berlin; Zdeňka Pilková und Ingeborg Allihn, „Benda (Familie)“, in: Ludwig Finscher (Hrsg.), Die Musik in Geschichte und Gegenwart. Zweite neubearbeitete Ausgabe, Personenteil 2, Kassel: Bärenreiter 1999, Sp. 1055-1074, hier Sp. 1067.

${ }^{21}$ Friedrich Wilhelm Gotter, Romeo und Julie: Ein Schauspiel mit Gesang in drey Aufzügen, Leipzig: Dyk 1779; Martin, „Shakespeare-Opern“ (wie Anm. 1), S. 7.
} 
führt 1836 in Magdeburg, hat im 19. Jahrhundert keine und - ungeachtet der Prominenz des Komponisten - auch im 20. Jahrhundert kaum einmal eine Wiederaufnahme gefunden. ${ }^{22}$

Andere Komponisten und Librettisten haben vornehmlich mit den Fehlschlägen ihrer Shakespeare-Opern in die Geschichtsbücher gefunden: Felix Mendelssohn-Bartholdy und Robert Schumann gaben die Arbeit an ihren Hamlet-Opern auf, Ludwig van Beethoven scheiterte an Macbeth. ${ }^{23}$ Zahlreiche andere Versuche teilten das Schicksal von Wagners Liebesverbot: Sie fanden keinen Anklang beim Publikum und gerieten ebenso schnell wie gründlich in Vergessenheit. Von vielen dieser Werke hat sich lediglich das Libretto erhalten, während die Musik verschollen ist, von manchen Werken gibt es gar nur eine vereinzelte Notizen, dass sie aufgeführt worden sind - das Verhältnis von der Quantität der Rezeption zu ihrer Dauerhaftigkeit ist im Hinblick auf die Shakespeareschen Dramentexte im deutschsprachigen Musiktheater also alles andere als günstig zu nennen, was übrigens aus unterschiedlichen Gründen für andere Sprachen ebenso gilt. Lässt man wiederum die Schauspielmusik mit all ihren Spielarten außer Acht, dauerte es selbst in England bis ins Jahr 1960, um Shakespeare ins Musiktheater bringen zu können: mit Benjamin Brittens A Midsummer Night's Dream, einer freilich meisterhaften Oper, die mit ihren musikalischen Verweisen auf andere Werke der Operntradition eine subtile Doppelbödigkeit entstehen lässt, die unmittelbar auf das kunstvolle Spiel im Spiel verweist, das auch Shakespeare so virtuos einsetzte.

Dieter Martin hat herausgestellt, dass im deutschsprachigen Raum zunächst jene Dramen Shakespeares für die Oper bearbeitet worden sind, die mit ihrem Stoff und ihren musikalischen Anteilen den Vorstellungen von der Oper an der Wende vom 18. zum 19. Jahrhundert am nächsten kommen: vor allem Die lustigen Weiber von Windsor, erstmals wohl von Karl Ditters von Dittersdorf als Die lustigen Weiber und der dicke Hanns im Jahr 1796 auf die Bühne des herzoglichen Theaters in niederschlesischen Oels gebracht, sowie Der Sturm und Ein Mittsommernachtstraum. ${ }^{24}$ Vom Mittsommernachtstraum allerdings hat zunächst keine Bearbeitung auf die deutschsprachige Opernbühne gefunden; zu denken ist allenfalls an Carl Maria von Webers Oberon aus dem Jahr 1826, dessen Uraufführung allerdings in englischer Sprache am Royal Opera House Covent Garden in London stattfand,

\footnotetext{
${ }^{22}$ Egon Voss, „Das Liebesverbot oder Die Novize von Palermo“, in: Carl Dahlhaus (Hrsg.), Pipers Enzyklopädie des Musiktheaters, Bd. 6, München: Piper 1997, S. 545-549, hier S. $548 f$.

23 Martin, „Shakespeare-Opern“ (wie Anm. 1), S. 1f. Martin, von dessen Beitrag auch die Zusammenstellung der Repertoirewerke abhängig ist, widmet Beethovens Hamlet-Libretto, das in der Beethoven-Forschung zuvor weitgehend übersehen worden ist, in diesem Aufsatz eine detaillierte Analyse.

${ }^{24}$ Martin, „Shakespeare-Opern“ (wie Anm. 1), S. 5.
} 
und der auch lediglich Motive aus dem Sommernachtstraum aufnimmt, ansonsten aber eine Handlung erzählt, die mit Shakespeares Vorlage nur wenig Verbindung hat. Die Oper beruht auf Wielands Übersetzung von James Robinson Planchés Oberon, die wiederum von William Sotheby ins Englische zurück übertragen worden ist. ${ }^{25}$ Auch Motive aus dem Sturm sind im Oberon auszumachen; der vermutlich erste Versuch, den Sturm zumindest nahe an der Shakespeareschen Vorlage auf die Opernbühne zu bringen, führt aber wiederum nach Magdeburg, dieses Mal allerdings in das Jahr 1779. Für seine alljährlich stattfindenden Winterkonzerte hatte der Magdeburger Musikdirektor Johann Heinrich Rolle das Libretto eines Magdeburger Predigers, Samuel Patzke, zur Vorlage genommen, das dieser wiederum der Wielandschen Übersetzung nachgebildet und zwei Arientexte auch wörtlich verwendet hatte. Unter dem Titel Prosper, oder die edelmüthige Rache erscheint das Drama Shakespeares allerdings erheblich gekürzt. Die Handlung ist in einem Akt mit 24 Nummern zusammengefasst und zeigt sich als regelmäßige Abfolge von Rezitativen und Arien bzw. Ensembles und Chorsätzen. Prosper wurde nachweislich nur vier Mal in Magdeburg gespielt, allerdings 1782 auch noch drei Mal in Berlin. ${ }^{26}$ Dennoch teilt Prosper das Schicksal der meisten ShakespeareAdaptionen dieser Zeit: Erhalten ist lediglich das Libretto. Es lässt sich damit auch nicht entscheiden, ob die Rezitative - wie bei Rolle sonst üblich - gesungen oder nach Art des Singspiels gesprochen worden sind. Immerhin gibt es aber eine anonyme zeitgenössische Rezension der Berliner Aufführungen. Über das Libretto und dessen Einrichtung äußert sich der Rezensent nur kurz, etwas ausführlicher aber über die Musik. Hier und da schimmere etwas hervor, so der Rezensent, was man bei freien Bearbeitungen dieser Art nicht zu sehen gewohnt sei. Die Musik sei im Ganzen gut, verfalle aber zuweilen in den Kirchenstil. ${ }^{27}$ Worauf sich der Rezensent dabei bezieht, lässt sich aufgrund des Fehlens einer Partitur bestenfalls mutmaßen: es könnte vielleicht die Kompositionstechnik der Chorsätze in diesem Werk sein. Was immer ihm aber die Komposition für das Musiktheater hat ungeeignet erscheinen lassen, dürfte an der Erfolglosigkeit dieses Versuchs indes ursächlich beteiligt gewesen sein.

Unter den Shakespeare-Dramen hat der Sturm um 1800 allerdings die stärkste Welle von Adaptionen für das deutschsprachige Musiktheater ausgelöst. Einschlägig ist hier die Version von Friedrich Hildebrand von Einsiedel und Friedrich Wilhelm Gotter, die unter dem Titel Die Geisterinsel mehrfach vertont worden ist.

\footnotetext{
${ }^{25}$ Carl Dahlhaus und Sieghart Döring, „Oberon or The Elf King’s Oath“, in: Carl Dahlhaus (Hrsg.), Pipers Enzyklopädie des Musiktheaters, Bd. 6, München: Piper 1997, S. 672-677, hier S. 672.

${ }^{26}$ Waczkat, Rolles musikalische Dramen (wie Anm. 5), S. 248f. und $410 \mathrm{f}$.

${ }^{27}$ Ebd., S. 249.
} 
Martin setzt die langjährige Entstehungszeit dieses Librettos zum Problem eines als desillusionierend erkannten Prosa-Dialogs zwischen den Gesängen und dem Versuch, den Text daher vollständig in Verse zu fassen, in Beziehung. ${ }^{28}$ Die eigentliche Fassung folgt dann jedoch wieder dem Typus des Singspiels mit gesprochenen Dialogen zwischen den Arien, wie er in jener Zeit auf der deutschsprachigen Opernbühne weit verbreitet ist. Das Libretto ist erstmals von Friedrich Fleischmann 1796 in Regensburg, danach 1798 mehr oder weniger gleichzeitig von Johann Friedrich Reichardt in Berlin und Johann Rudolf Zumsteeg in Stuttgart vertont worden. ${ }^{29}$ Während Fleischmanns Vertonung kein und Reichardts nur geringer Erfolg beschieden war, war Zumsteegs Oper für rund zwei Jahrzehnte gut im deutschsprachigen Raum vertreten. Nach der Inszenierung in Mannheim 1817 verschwindet jedoch auch dieses Werk wieder aus dem Repertoire. ${ }^{30}$ Einer Vertonung von Peter von Winter, die einer Librettofassung des Sturm von Franz Xaver Caspar folgt, ist nach ihrer Uraufführung 1798 im Münchner Residenztheater gleichfalls keine spätere Wiederaufnahme gefolgt.

Und so sind es letztlich nur zwei Adaptionen Shakespearescher Dramen für die deutschsprachige Opernbühne, die sich längere Zeit in den Spielplänen behaupten konnten: Otto Nicolais Die lustigen Weiber von Windsor und Hermann Goetz' Der Widerspenstigen Zähmung. Beide Opern bestätigen indes einmal mehr, ungeachtet der dazwischen liegenden Jahrzehnte, das eingangs skizzierte Umfeld der bildungsbürgerlichen Selbstvergewisserung. Nicolai, geboren 1810 in Königsberg, studierte seit 1827 in Berlin am Königlichen Institut für Kirchenmusik bei Carl Friedrich Zelter; er war Mitglied der Berliner Sing-Akademie und hat somit 1829 mutmaßlicherweise an der legendären Aufführung der Matthäus-Passion Johann Sebastian Bachs durch Mendelssohn mitgewirkt. Nachdem er für einige Zeit eine Organistenstelle an der Preußischen Gesandtschaftskapelle in Rom inne hatte, wechselte er 1837 kurzfristig als Kapellmeister an das Wiener Kärntnertortheater, von wo aus er aber schon im folgenden Jahr wiederum nach Rom ging. 1841 kehrte er erneut nach Wien zurück auf die Stelle des Ersten Kapellmeisters an der Wiener Hofoper. 1847 schließlich folgte ein Ruf als Kapellmeister an das Königliche Theater in

\footnotetext{
${ }^{28}$ Martin, „Shakespeare-Opern“ (wie Anm. 1), S. 6.

${ }^{29}$ Christoph-Hellmut Mahling, „Die Geisterinsel“, in: Carl Dahlhaus (Hrsg.), Pipers Enzyklopädie des Musiktheaters, Bd. 6, München: Piper 1997, S. 817f, hier S. 817.

${ }^{30}$ Christoph-Hellmut Mahling, „Die Geisterinsel“, in: Carl Dahlhaus (Hrsg.), Pipers Enzyklopädie des Musiktheaters, Bd. 6, München: Piper 1997, S. 817f, hier S. 818.
} 
Berlin, wo ihm kurze Zeit darauf auch die Position des Dirigenten des Königlichen Domchores übertragen wurde. ${ }^{31}$

Nicolai starb schon zwei Jahre darauf und überlebte die Uraufführung seiner Oper Die lustigen Weiber von Windsor am 9. März 1849 um gerade einmal acht Wochen. Nicolai hatte sich schon früher für diesen Stoff interessiert und ein geeignetes Libretto von dem Kasseler Schriftsteller Jakob Hoffmeister erbeten, das dieser jedoch nicht liefern konnte oder wollte, woraufhin Nicolai den Wiener Dramatiker Salomon Hermann Mosenthal mit der Ausarbeitung dieses Librettos beauftragte. ${ }^{32}$ Offenbar in enger wechselseitiger Abstimmung mit Nicolai hat Mosenthal einen Text erarbeitet, der in der Tradition des deutschen Singspiels mit gesprochenen Dialogen steht und gegenüber der Vorlage nicht nur deutlich gekürzt, sondern dabei auch auf die komödiantischen Elemente zugespitzt ist. Daraus resultiert unter anderem eine Verlagerung des Gewichts von den männlichen auf die weiblichen Hauptrollen: In den Händen der Frauen Fluth und Reich laufen die Fäden des Spiels zusammen, und Anna, bei Shakespeare lediglich episodenhaft gezeichnet, wird zu einer Charakterfigur, die ihr Schicksal selbst in die Hand nimmt.

Nicolais Oper ist ausgezeichnet von einer Vielzahl musikalischer Anspielungen und Doppelbödigkeiten, die in der späteren Rezeption häufig übersehen worden sind. So zitiert Nicolai musikalisch aus Webers Oberon und Mendelssohns Schauspielmusik zum Sommernachtstraum; in ironischer Brechung erscheinen außerdem Reminiszenzen an Heinrich Marschners Oper Der Vampir und Wagners Der fliegende Holländer. ${ }^{33}$ Und eine veritable musikalische Parodie ist das Duett von Falstaff und Fluth im zweiten Akt der Oper, das in einem bewusst operntümelnden Stil gehalten ist. Nicolai überführt diesen Dialog in ein übertrieben pathetisches Accompagnato-Rezitativ, dessen Schwere ein Element brachialer Komik ist.

Mit Der Widerspenstigen Zähmung von Hermann Goetz ist 1874 der Schlusspunkt der Shakespeare-Rezeption im deutschsprachigen Musiktheater des 19. Jahrhunderts erreicht. Wie Nicolai stammt auch der 1840 geborene Goetz aus Königsberg, wo er im bürgerlichen Umfeld einer Kaufmannsfamilie aufwuchs. Ein begonnenes Mathematikstudium brach er 1860 zugunsten eines Studiums am

\footnotetext{
31 Ulrich Konrad, „Otto Nicolai“, in: Ludwig Finscher (Hrsg.), Die Musik in Geschichte und Gegenwart. Zweite neubearbeitete Ausgabe, Personenteil 12, Kassel: Bärenreiter 2004, Sp. 1052-1057, hier Sp. 1052-1054.

32 Robert Didion, „Die lustigen Weiber von Windsor“, in: Carl Dahlhaus (Hrsg.), Pipers Enzyklopädie des Musiktheaters, Bd. 4, München: Piper 1991, S. 423-426, hier S. 423.

${ }^{33}$ Robert Didion, „Die lustigen Weiber von Windsor“, in: Carl Dahlhaus (Hrsg.), Pipers Enzyklopädie des Musiktheaters, Bd. 4, München: Piper 1991, S. 423-426, hier S. 425.
} 
Stern'schen Konservatorium in Berlin ab. 1864 erhielt Goetz eine Anstellung als Organist an der Stadtkirche von Winterthur, wo er vielseitigen musikalischen Tätigkeiten als Pianist, Dirigent, Organist und Klavierlehrer nachging. Eine Lungenerkrankung führte schon 1876 zu seinem Tod. ${ }^{34}$

Der Widerspenstigen Zähmung, die dritte und letzte vollständige von vier Opern Goetz' wurde am 11. Oktober 1874 in Mannheim aufgeführt, nachdem Goetz schon seit 1868 daran gearbeitet hatte. Das Libretto stammt zu etwa einem Drittel von Goetz selber, zum größeren Teil aber von dem Schweizer Schriftsteller Joseph Victor Widmann, einem studierten Theologen und Direktor einer Mädchenschule in Bern. ${ }^{35}$ Gegenüber der Vorlage Shakespeares, mit der Goetz und Widmann recht frei verfahren, sind die Charaktere einseitiger gezeichnet. Der Geschlossenheit der Opernhandlung kommt das entgegen, wenngleich auf Kosten des subtilen Humors, der in dieser Bearbeitung letztlich doch etwas hölzern und biedermeierlich anmutet, was vielleicht auch einen Anteil daran hat, dass diese bis zur Mitte des 20. Jahrhunderts recht häufig gespielte Oper gegenwärtig nur noch selten in den Spielplänen erscheint.

Bei aller Begeisterung für seine Dramen sind die Werke Shakespeares auf der deutschsprachigen Opernbühne somit doch ein Fremdkörper geblieben. Zum Einen ist dies wohl auf die Einsicht der Komponisten und Librettisten zurückzuführen, dass sich Shakespeares Stücke nicht oder nur unter großen Einbußen vom Sprech- ins Musiktheater überführen lassen, zum Anderen dürfte auch das Überangebot an äußerst erfolgreichen Opern in italienischer, teils auch französischer Sprache zu einer gewissen Stagnation geführt haben. Und insbesondere haben Gioacchino Rossinis Otello und mehr noch die drei Shakespeareopern Giuseppe Verdis, Macbeth, Otello und Falstaff, in italienischer Sprache exemplarisch Möglichkeiten aufgezeigt, denen auf der deutschsprachigen Opernbühne nicht angemessen zu begegnen war. Mit dem Erfolgsmodell der italienischen Oper, die schon im 17. Jahrhundert zu einem erstrangigen Exportgut in alle europäischen Länder geworden ist, wollte und konnte man im deutschsprachigen Raum nicht konkurrieren. Die Entwicklung von den Singspielen des 18. und 19. Jahrhunderts über Albert Lortzings Spielopern hin zu den Musikdramen Richard Wagners ist vielmehr eine, die man mit Sieghart Döhring und Sabine Henze-Döhring auch als

\footnotetext{
${ }^{34}$ Matthias Wiegandt, „Hermann Goetz“, in: Ludwig Finscher (Hrsg.), Die Musik in Geschichte und Gegenwart. Zweite neubearbeitete Ausgabe, Personenteil 7, Kassel: Bärenreiter 2002, Sp. 1214-1217, hier Sp. $1214 f$.

35 Klaus Kropfinger, „Der Widerspenstigen Zähmung“, in: Carl Dahlhaus (Hrsg.), Pipers Enzyklopädie des Musiktheaters, Bd. 2, München: Piper 1987, S. 472-475, hier S..
} 
„Krise“ bezeichnen kann $^{36}$ und die erst in Wagners Konzeptionen eine eindeutige Richtung gefunden hat: eine, die vom Kontext bürgerlichen Strebens nach geistiger Bildung erkennbar unabhängig ist.

\section{Bibliographie}

Benda, Jiři Antonín, „Klavierauszug von Romeo und Julie, einer Oper in drey Akten, in Musik gesetzt" (Leipzig: Dyk 1778), Die Musik in Geschichte und Gegenwart. Zweite neubearbeitete Ausgabe, Personenteil 2, Hg. Ludwig Finscher, Kassel: Bärenreiter 1999, 1055-1074.

Gerstenberg, Heinrich Wilhelm von, „Phantasie von C. P. E. Bach, mit doppelt unterlegtem Text von Gerstenberg", Flora. Erste Sammlung enthaltend Compositionen für Gesang und Clavier, Hg. Carl Friedrich Cramer, Kiel: [Selbstverlag], 1787.

Dahlhaus, Carl und Döring, Sieghart, „Oberon or The Elf King's Oath“, Pipers Enzyklopädie des Musiktheaters, Bd. 6, Hg. Carl Dahlhaus, München: Piper, 1997. 672-677.

Didion, Robert, „Die lustigen Weiber von Windsor“, Pipers Enzyklopädie des Musiktheaters, Bd. 4, Hg. Carl Dahlhaus, München: Piper 1991.

Döhring, Sieghart und Henze-Döhring, Sabine. Oper und Musikdrama im 19. Jahrhundert, Handbuch der musikalischen Gattungen, 13, Laaber: Laaber Verlag, 1997.

Draheim, Joachim, „Shakespeare“, Die Musik in Geschichte und Gegenwart. Zweite neubearbeitete Ausgabe, Hg. Ludwig Finscher, Personenteil 15, Kassel:

Bärenreiter 2006.

Edler, Arnfried, „Carl Philipp Emanuel Bachs Wirkung auf das Musikleben seiner Zeit", Carl Philipp Emanuel Bach. Musik und Literatur in Norddeutschland. Schriften der Schleswig-Holsteinischen Landesbibliothek, 4, Heide: Westholsteinische Verlagsanstalt 1988, 20-39.

Gotter, Friedrich Wilhelm. Romeo und Julie: Ein Schauspiel mit Gesang in drey Aufzügen. Leipzig: Dyk 1779.

36 Sieghart Döhring und Sabine Henze-Döhring, Oper und Musikdrama im 19. Jahrhundert (Handbuch der musikalischen Gattungen; 13), Laaber: Laaber Verlag 1997, S. 11 und S. 91-143. 
Jacobshagen, Arnold, „Opernkritik und Opern-,Reform”, Die Oper im 18. Jahrhundert. Hg. Herbert Schneider und Siegfried Wiesend, Handbuch der musikalischen Gattungen, 12, Laaber: Laaber-Verlag 2001, 74-84.

Kaschuba, Wolfgang, „Deutsche Bürgerlichkeit nach 1800. Kultur als symbolische Praxis", Bürgertum im 19. Jahrhundert. Deutschland im europäischen Vergleich, Hg. Jürgen Kocka, Bd. II: Wirtschaftsbürger und Bildungsbürger, Göttingen: Vandenhoeck und Ruprecht 1995, 92-127.

Konrad, Ulrich, „Otto Nicolai“, Die Musik in Geschichte und Gegenwart. Zweite neubearbeitete Ausgabe, Personenteil 12, Hg. Ludwig Finscher, Kassel: Bärenreiter 2004.

Kropfinger, Klaus, „Der Widerspenstigen Zähmung“, Pipers Enzyklopädie des Musiktheaters, Bd. 2, Hg. Carl Dahlhaus, München: Piper, 1987.

Leopold, Silke und Zimmermann, Michael, „Opernreformen“, Die Musik des 18. Jahrhunderts, Hg. Carl Dahlhaus, Neues Handbuch der Musikwissenschaft; 5 , Laaber: Laaber-Verlag 1985, 239-253.

Lütteken, Laurenz. Das Monologische als Denkform in der Musik zwischen 1760 und 1785. Wolfenbütteler Studien zur Aufklärung, 24, Tübingen: Niemeyer 1998.

Mahling, Christoph-Hellmut, „Die Geisterinsel“, Pipers Enzyklopädie des Musiktheaters, Bd. 6, Hg. Carl Dahlhaus, München: Piper, 1997.

Martin, Dieter, „Deutsche Shakespeare-Opern um 1800 (08.10.2005)“, Goethezeitportal, http://www.goethezeitportal.de/db/wiss/epoche/martin_shakespeare_opern.pdf (zuletzt aufgerufen am 30.05.2015).

Sulzer, Johann Georg. Allgemeine Theorie der schönen Künste, 2te Auflage. Leipzig: Weidmann 1792-1794, Bd. III.

Voss, Egon, „Das Liebesverbot oder Die Novize von Palermo“, Pipers Enzyklopädie des Musiktheaters, Bd. 6, Hg. Carl Dahlhaus, München: Piper, 1997. 545-549.

Waczkat, Andreas. Johann Heinrich Rolles musikalische Dramen. Theorie, Werkbestand und Überlieferung einer Gattung im Kontext bürgerlicher Empfindsamkeit, Beeskow: ortus 2007

Wiegandt, Matthias, „Hermann Goetz“, Die Musik in Geschichte und Gegenwart. Zweite neubearbeitete Ausgabe, Personenteil 7, Hg. Ludwig Finscher, Kassel: Bärenreiter 2002. 